\title{
Radar distance measurements in circular waveguides involving intermodal dispersion effects
}

\author{
ECKHARD DENICKE, GUNNAR ARMBRECHT AND ILONA ROLFES
}

\begin{abstract}
This contribution deals with guided radar distance measurements in the field of industrial tank level control. The aim is to achieve a submillimeter gauging accuracy even when conducting the measurement within a highly dispersive environment of large and thus overmoded circular waveguides. Normally, multimode propagation causes a decrease in measurement precision. Therefore, the effects of intermodal dispersion are fundamentally reviewed and, based on these results, a correlationbased signal processing method is presented. This method is able to exploit the otherwise parasitic dispersion effects to enhance the measurement precision even in constellation with a simple waveguide transition or antenna, respectively. Furthermore, considerations on the mode variety and its influence on the signal complexity as well as investigations on the technique's reliability and accuracy are presented. Measurement results in a frequency range of 8.5-10.5 GHz are provided for three different kinds of waveguide transitions proving the capability of the method.
\end{abstract}

Keywords: Radar distance measurement, Level measurement, Radar signal processing, Correlation, Overmoded circular waveguides, Multimode waveguides, Intermodal dispersion

Received 1 December 2009; Revised 29 April 2010; first published online 25 June 2010

\section{INTRDDUCTION}

In the process instrumentation industry, radar techniques are commonly applied for high-precision distance measurements in free-space applications, e.g. for tank level probing of liquids [1]. In this contribution, however, the measurement is conducted in large tank-mounted metal tubes, often called still pipes or stilling wells, acting as overmoded circular waveguides, as illustrated in Fig. 1. In this case, the waveguide's feeding section, represented by the utilized antenna or by the respective transition between the mono-moded feeding waveguide and the overmoded metal tube, often leads to the excitation and consequently, the propagation of higher-order modes within the system. To explain the consequences of this matter, Fig. 1 highlights two cases of different waveguide transitions applied in a tank system.

On the left-hand side, a long conical horn is used, providing a smooth transition between the different waveguide diameters, which thus leads to a mainly mode-preserving transmission of the used fundamental mode $H_{11}$ to the overmoded waveguide. The electric field distribution of the sole fundamental mode, obtained from a commercial $3 \mathrm{D}$ FIT field simulator (CST MICROWAVE STUDIO, Vers. 2009), is depicted within the left tube as a snap-shot for one point of time, propagating as a gaussian pulse in the time domain ( $20 \%$ fractional bandwidth). Due to the large tube diameter, the mode-inherent (chromatic) dispersion of the $H_{11}$ mode

Institute of Radiofrequency and Microwave Engineering, Leibniz Universität Hannover, Appelstr. 9A, 30167 Hannover, Germany.

Corresponding author:

E. Denicke

Email: denicke@hft.uni-hannover.de may be neglected [2], so the measurement device is able to receive an almost undistorted pulse returning from the surface of the medium.

The metal tube on the right-hand side is equipped with a much shorter conical horn, representative for less costintensive transitions that are intentionally simple and more compact. These transitions are often requested by industry. As a result, a multitude of modes is excited due to the horn's precipitous flare angles, which thus propagates within the metal tube. Hence, because of the different propagation velocities of each mode's signal portion, the planar phase fronts of the pulse are distorted in comparison to the left-hand side. Furthermore, the signal energy is more and more spread within the metal tube with increasing propagation distance. This leads to nonlinear phase distortions in frequency domain and to a deteriorated pulse shape in time domain, respectively.

Due to this effect the gauging accuracy is significantly decreased, when using conventional free-space optimized signal processing, like pulse-based barycenter computation in time domain [3]. To sum up, retaining conventional signal processing demands for significant effort for the waveguide transition to avoid spurious mode excitation, especially when complying with compact geometrical constraints [4].

Therefore, this contribution deals with a different approach to achieve the desired submillimeter measurement precision (see also [5]). To understand how the method works, initially the influences of multimode propagation, i.e. the two dominating effects of intermodal dispersion and their resultant pulse shape distortion are described in detail in Section II. Subsequently, the core of the proposed measurement method is introduced in Section III as an alternative correlation-based signal processing algorithm, exploiting the 


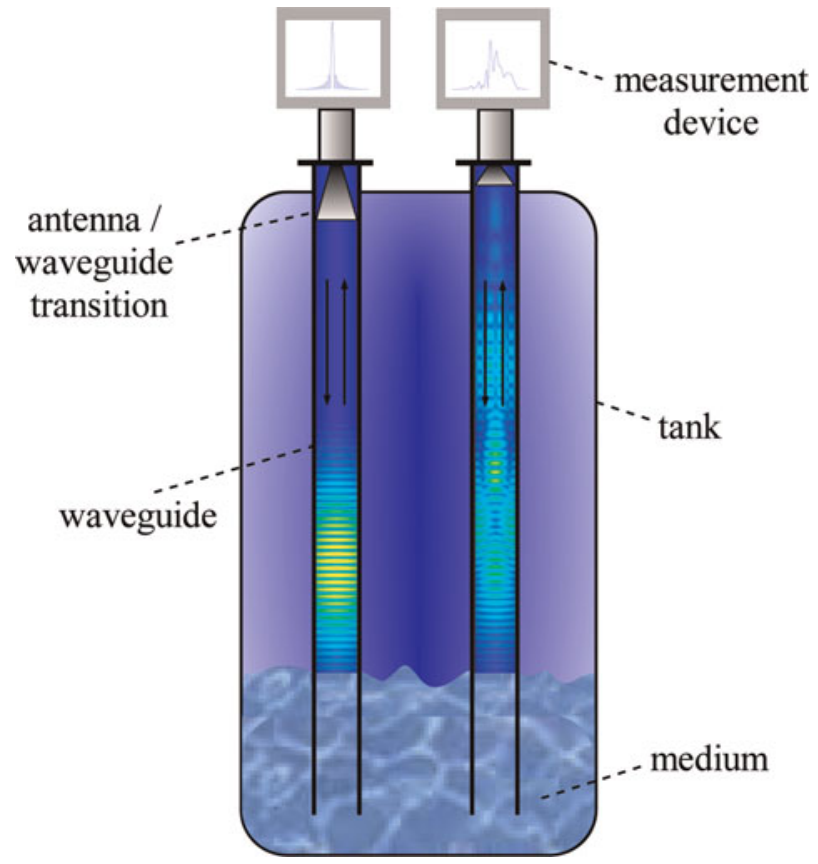

Fig. 1. Guided radar distance measurement in an industrial tank environment conducted with two different waveguide transitions.

multimode signal distortion. Section III.A presents, considerations on the mode variety and its influence on the signal complexity, followed in Section III.B by investigations on the technique's reliability and accuracy. Finally, the measurement results in Section IV indicate the capability of the proposed method.

\section{EFFECTS DF INTERMDDAL DISPERSIDN}

In this section, the basic effects of multimode propagation on pulse shape deformation are investigated. Based on the theoretical fundamentals of analytical waveguide equations accounting for the mode-dependent propagation behavior inside the metal tube, a MATLAB-based waveguide simulator is utilized in conjunction with an analytical model of a waveguide transition to synthesize multimode propagation scenarios [2].

Exemplarily, a scenario with only one parasitic mode $E_{11}$ with a transmission level of $-3 \mathrm{~dB}$ for the scattering parameter $\left|S_{2\left(E_{11}\right),{ }_{1}\left(H_{11}\right)}\right|$ is assumed. For this case, various impulse response envelopes, each with a normalized time axis and the main pulse package at o ns, are depicted in Fig. 2(a) for an increasing reflector distance in the interval of $l_{\text {refl }}=[0.2 \ldots 0.7 \mathrm{~m}]$. The curves are obtained from the inverse Fourier transform of the system's reflection coefficient in the frequency domain with a bandwidth from 8.5 to 10.5 $\mathrm{GHz}$ for an inner tube diameter of $d_{s p}=80 \mathrm{~mm}$. As a basic principle, waveguide transitions, when supposed to be loss-free multiport devices featuring independent ports for each excited mode, cannot be matched for every single port, if multimode excitation is present [2]. As a result, modedependent multiple reflection cycles arise inside the tube, that generate pulse replicas, which may exceed the peak amplitudes of the first main reflection pulse. This evolves from a phenomenon comparable to the "mode beating" effect known from the theory of optical transmission lines [6]. Consequently, a conventional barycentric processing algorithm could unlatch due to wrong detection on pulse maxima of replicas.

An oscillating amplitude can be observed here for each pulse package due to constructive or destructive interferences between the different modes' signal portions. This is caused by phase differences due to unequal round trip times for the metal tube, after converting back to the fundamental mode in the mono-moded feeding section of the transition. Possible constellations for erroneous detection on the first pulse replica are marked. The period length of the oscillation or the distance between two reflector positions $l_{\text {refl }}$ with maximal amplitudes, respectively, is approximated by the beat length (compare [7]):

$$
l_{\text {beat }\left(H_{11}, E_{11}\right)}=\frac{\pi}{\beta_{H_{11}}-\beta_{E_{11}}} .
$$

Herein the variable $\beta$ denotes the phase constant of the particular mode at the center frequency.

Referring to the one parasitic mode scenario again, Fig. 2(b) depicts the impulse response envelopes for a larger reflector interval of $l_{\text {refl }}=[0 . .5 \mathrm{~m}]$, whereas pulse replicas are explicitly excluded for the purpose of clarity.

Evidently, with the present spurious $E_{11}$ mode, the obtained package consists of two pulses, with the $E_{11}$ pulse being delayed, owing to its higher cut-off frequency and consequently its lower propagation velocity. This leads to a temporal walk-off between the different modes' signal portions, causing a barycenter distortion of the pulse package. Finally, the walk-off results in a pulse breakup with increasing reflector distance $l_{\text {refl }}[8]$. Hence, for large reflector distances the intensity of the interferences and thus the beating of the pulse package amplitude also decreases. Additionally, the shape of the separated $E_{11}$ pulse becomes more and more deformed due to its increased chromatic dispersion in comparison to the fundamental mode.

\section{A CDRRELATION-BASED SIGNAL PRDCESSING ALGORITHM}

Being aware of the intermodal dispersion effects, a different approach for a correlation-based signal processing algorithm is derived in this section. As a paradigm shift, the observed dispersion effects are now exploited instead of being usually considered as parasitic. Accordingly, the idea of the proposed algorithm is to evaluate the shape of multimode-distorted impulse responses, whose energy is spread in the time domain in a unique way being unambiguously associated with every distinct reflector distance. In this case, the analytically describable environment in the metal tube is utilized to generate synthetic reference signals for various reflector distances representing varying medium levels. The developed simulation model [4] rebuilds the measurement setup incorporating a metal tube with a movable planar sliding short acting as a reflector with a maximal distance of $l_{\text {refl,max }}=$ $1.229 \mathrm{~m}$ (Fig. 3) to compare signals from the measurement with simulated reference signals via correlation.

Conventionally, correlation-based algorithms are often used for echo detection in radar applications, where a measured signal is correlated with the transmitted signal, 


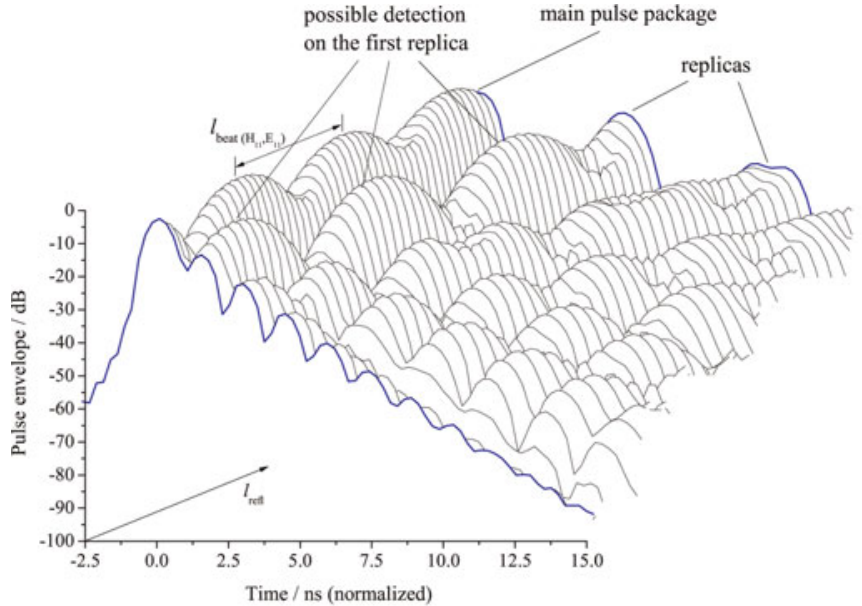

(a)

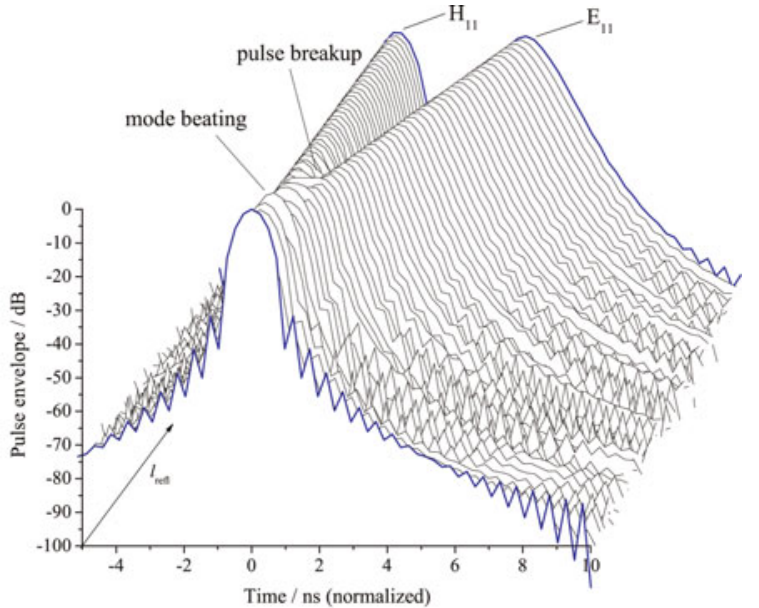

(b)

Fig. 2. The dominating effects of intermodal dispersion: (a) mode beating and multiple replicas and (b) pulse breakup within the main pulse package.

being equivalent to matched filtering [9]. In the presence of intermodal dispersion, correlation with the transmitted signal is meaningless due to the distance-dependent shape of the received signal. Consequently, in this case the measured signal or the complex envelope of the impulse response $g_{c e, \text { meas }}(t)$, respectively, is rather correlated with a table of simulated reference complex envelopes $g_{c e}\left(t, l_{\text {ref, tab }}\right)$ for every feasible reflector distance $l_{\text {reff,tab }}$ in a desired step width $\Delta l_{\text {refl, } t a b}$, specified here without loss of generality in a continuous time notation. This is equivalent to the application of various appropriate matched filters for each value of $l_{\text {refl, tab }}$. Moreover, reference signals could also be taken from reference measurements with variable distances, incorporating e.g. the sliding short (Fig. 3(c)).

The cross-correlation function $R\left(\sigma, l_{\text {refl, tab }}\right)$, as a quantity for the similarity between two functions or rather two energy signals here, is defined in accordance to [10], as follows:

$$
R\left(\sigma, l_{\text {refl,tab }}\right)=\int_{-\infty}^{+\infty} \bar{g}_{c e}\left(t+\sigma, l_{\text {refl,tab }}\right) \cdot \bar{g}_{c e, \text { meas }}^{*}(t) d t
$$

with

$$
\bar{g}_{c e}(t)=\sqrt{\frac{1}{\int_{-\infty}^{+\infty}\left|g_{c e}(t)\right|^{2} d t}} \cdot g_{c e}(t)
$$

$$
\Leftrightarrow \int_{-\infty}^{+\infty}\left|\bar{g}_{c e}(t)\right|^{2} d t=1
$$

Because magnitudes have to be comparable between every computed correlation, each complex envelope is normalized to a signal energy of unity. At the global maximum of the magnitude of $R\left(\sigma, l_{\text {refl,tab }}\right)$ over all $l_{\text {reff,tab }}$ the particular reference distance $l_{\text {refl, tab }}$ is chosen as the measured distance $l_{\text {refl,meas }}$. In case of an excitation port of the simulation coinciding with the calibration plane in the measurement, maximal similarity is expected for the quasi-autocorrelation, i.e. when the distance $l_{\text {reff, tab }}$ from the reference table matches with the actual reflector distance $l_{\text {refl }}$ in the setup. For this reason, the global maximum of $\left|R\left(\sigma, l_{\text {refl, tab }}\right)\right| \forall l_{\text {refl, tab }}$ is anticipated to be at $\sigma=0$, exhibiting a value of unity in case of perfect conformance between the reference and the measurement signal, corresponding to the signal energy. For all non-matching $l_{\text {refl,tab }}$ the correlation magnitude at $\sigma=0$ is smaller. This fact renders possible to avoid the calculation of the entire correlation function over all $\sigma$.

Accordingly, the measured distance $l_{\text {reft,meas }}$ is defined as

$$
\begin{aligned}
l_{\text {reff,meas }}= & \left\{l_{\text {reff,tab }} \in\left\{0, \Delta l_{\text {refl,tab }}, \ldots, l_{\text {refl,max }}\right\} \mid \ldots\right. \\
& \left.\ldots\left|R\left(\sigma=0, l_{\text {refl,tab }}\right)\right| \text { maximal }\right\} .
\end{aligned}
$$

The procedure is summarized in Fig. 4 as a block diagram.

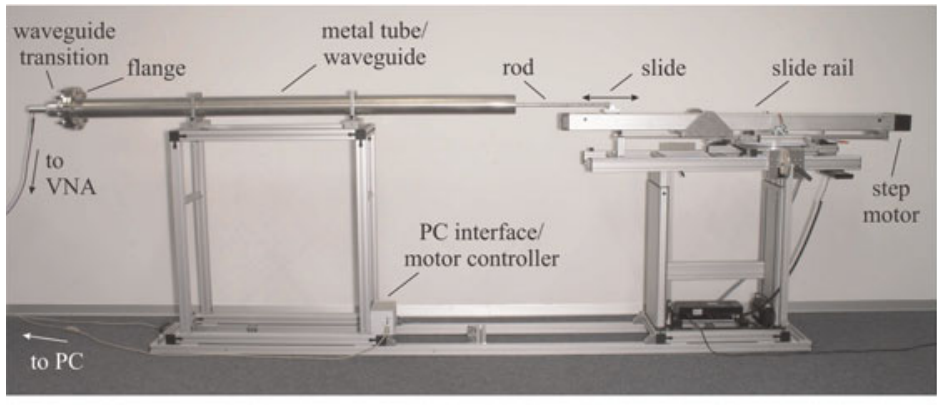

(a)

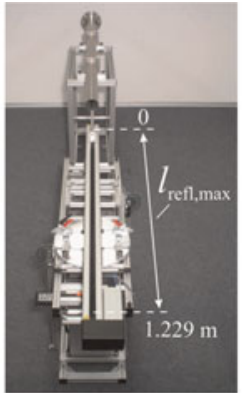

(b)

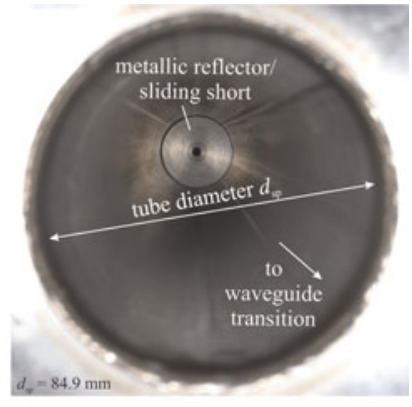

(c)

Fig. 3. Measurement setup: (a) side view (b) top view and, (c) sliding short inside the tube. 


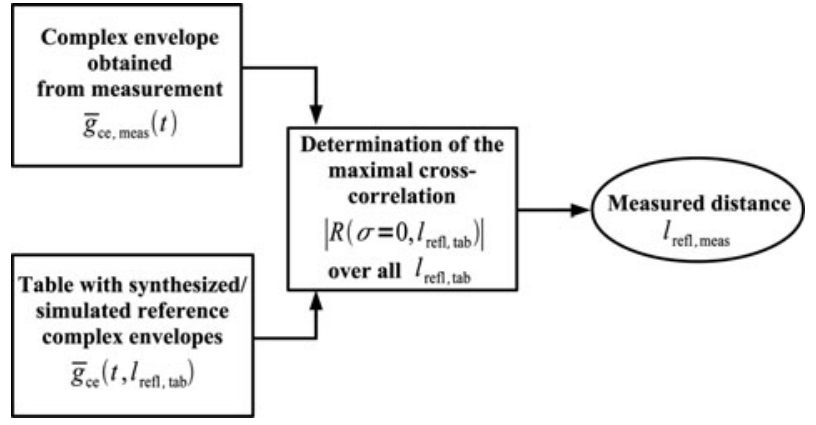

Fig. 4. Block diagram of the correlation-based method.

\section{A. Considerations on mode variety and signal complexity}

As the proposed method presented in this article depends on the intermodal dispersion effects due to the propagation behavior of waveguide modes in a metal tube, in this section the influence of the mode variety on the signal shape complexity and its impact on the algorithm are evaluated fundamentally. The investigations are based on the analytical model of a waveguide transition in the frequency range of 8.510.5 GHz (compare Section II and [2]), connected to a waveguide with the inner diameter of $d_{s p}=84.9 \mathrm{~mm}$ as found in the measurement setup (Fig. 3(c)).

Theoretically, such an overmoded cross-section possesses a high amount of propagable eigenmodes in the used frequency range. But if the feeding transition of the metal tube is excited by the fundamental mode $H_{11}$ and its taper is axially straight with circular symmetry, the only excited modes in the large cross-section are of the $E / H_{1 p}$-type [11]. Consequently, under the proposed constraints the dominant modes in the metal tube are $H_{11}, E_{11}, H_{12}, E_{11}$, and $H_{13}$, exhibiting cut-off frequencies $f_{c}$ as listed in Table 1 . The fifth dominant mode $H_{13}$ is not propagable within the whole frequency range, so for the investigations four simulated gauging scenarios with a number of solely one to four modes are defined to generate signals with different signal complexity as described in the following. In contrast to [4], no mode is considered as parasitic, so all modes are covered equally by splitting the feeding power to identical parts, leading to excitation levels of 100,50 (compare Section II), 33.3, and 25\% for each mode within the particular scenario in relation to the feeding power (summarized in Table 1). These scenarios represent different types of waveguide transitions featuring distinctive levels of higherorder mode excitation (compare manufactured transitions in Section IV).

To analyze this matter, the magnitudes of the impulse response envelopes for all four scenarios are compared in Fig. 5 at three reflector distances $l_{\text {ref }}=0.2,0.6$, and $1.0 \mathrm{~m}$. In general, each signal consists of as many modal sub signals or signal portions as it exhibits excited modes, each propagating at its own velocity, leading to the aforementioned effects of intermodal dispersion (Section II).

For a small reflector distance of e.g. $l_{\text {refl }}=0.2 \mathrm{~m}$ (Fig. $5(\mathrm{a})$ ) the individual signal portions are still merged in the case of two or more modes, but a slight position and shape deterioration of the main pulse package is already visible. Additionally, the amplitudes of all pulse packages differ among the four scenarios due to the particular mode
Table 1. Simulated gauging scenarios; cut-off frequencies of the dominant modes for $d_{s p}=84.9 \mathrm{~mm}$.

\begin{tabular}{llllll}
\hline & $\boldsymbol{H}_{\mathbf{1 1}}$ & $\boldsymbol{E}_{\mathbf{1 1}}$ & $\boldsymbol{H}_{\mathbf{1 2}}$ & $\boldsymbol{E}_{\mathbf{1 2}}$ & $\boldsymbol{H}_{\mathbf{1 3}}$ \\
\hline 1 mode $(100 \%, \mathrm{odB})$ & $\mathrm{X}$ & - & - & - & - \\
$2 \operatorname{modes}(50 \%,-3 \mathrm{~dB})$ & $\mathrm{X}$ & $\mathrm{X}$ & - & - & - \\
3 modes $(33.3 \%,-4.8 \mathrm{~dB})$ & $\mathrm{X}$ & $\mathrm{X}$ & $\mathrm{X}$ & - & - \\
$4 \operatorname{modes}(25 \%,-6 \mathrm{~dB})$ & $\mathrm{X}$ & $\mathrm{X}$ & $\mathrm{X}$ & $\mathrm{X}$ & - \\
$f_{c} / \mathrm{GHz}$ & 2.07 & 4.31 & 5.99 & 7.89 & 9.59 \\
\hline
\end{tabular}

excitation levels and due to the mode beating effect involving different modes. The first replica of the main pulse package for example, caused by a second reflection of the signal at the short, is observable solely in case of three and four modes at twice the time $\left(\approx_{3} \mathrm{~ns}\right)$ and is thus obviously suppressed by mode beating at this reflector position in case of two modes.

For a larger reflector distance of $l_{\text {refl }}=0.6 \mathrm{~m}$ (Fig. $\left.5(\mathrm{~b})\right)$ the separation of some of the individual mode pulses is already observable and the main pulse package is broken up. Generally, modes with higher cut-off frequency or smaller velocity of propagation, respectively, separate first with increasing reflector distance.

Finally, for a distance of $l_{\text {refl }}=1.0 \mathrm{~m}$ (Fig. 5(c)) the specific mode pulses are clearly dividable and marked. According to the excitation levels of each scenario (Table 1) the amplitude of all pulses decreases with increasing number of modes. A decaying amplitude from modes with a low to a high cut-off frequency due to mode-inherent chromatic dispersion is also visible. Thus, the $E_{12}$ exhibits the smallest amplitude and the strongest deformed shape. In the one mode scenario no intermodal dispersion and no replicas are present (see also [2]), whereas the pulse of the $H_{11}$ with its marginal chromatic dispersion is almost undeformed and only proportionally shifted in time with the corresponding reflector distance.

Summing up, as the number of modes increases, the signal shape becomes generally more and more complex and unambiguously associated with the particular reflector distance, because more distinctive individual pulses and thus more interferences arise, representing the intermodal dispersions effects between multiple modes.

To investigate now the influence of the signal complexity on the proposed method, the correlation-based algorithm is used in the four simulation scenarios to calculate the absolute values of the cross-correlation function $\left|R\left(\sigma=0, l_{\text {reff }}\right)\right|$ for an exemplary reflector distance of $l_{\text {refl }}=1.0 \mathrm{~m}$ (compare envelope in Fig. 5(c)), which is assumed to be measured. As depicted in Fig. 6, the correlation is conducted in dependence of all reference distances from the table $l_{\text {refl,tab }}=\left[0 \ldots, l_{\text {refl, } \max }\right]$, leading to a horizontal curve resolution of $\Delta l_{\text {refl,tab }}$. Thus, for a reference distance $l_{\text {ref, }, \text { tab }}$ matching with the actual distance $l_{\text {refl }}$ accounting for the autocorrelation, the magnitude is high and exhibits the theoretical maximum of $\left|R\left(\sigma=0, l_{\text {ref, tab }}\right)=1\right|$ (owing to the energy normalization). In case of one mode the curve is almost symmetrical to $l_{\text {refltab }}=1.0 \mathrm{~m}$ due to the corresponding time signals being only shifted in time over the distance $l_{\text {refftab }}$ but little deformed in shape.

Moreover, with increasing number of modes, the obtained main peak width becomes narrower, i.e. correlation with adjacent reference signals in the region of the adequate one declines faster, which is caused by more intermodal dispersion and thus more signal shape deformation per distance; even the side lobes become narrower. 


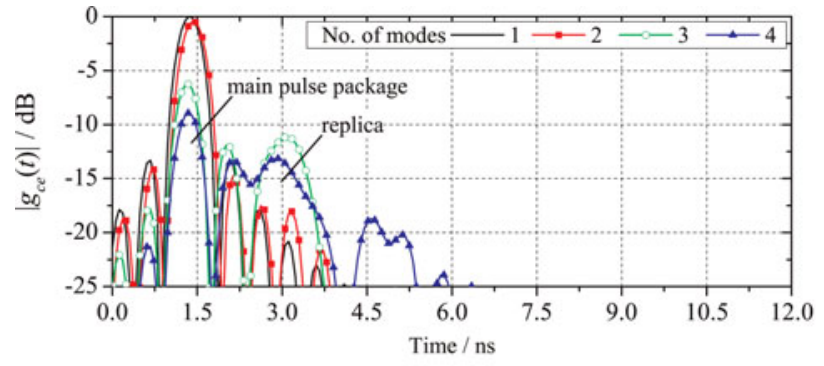

(a)

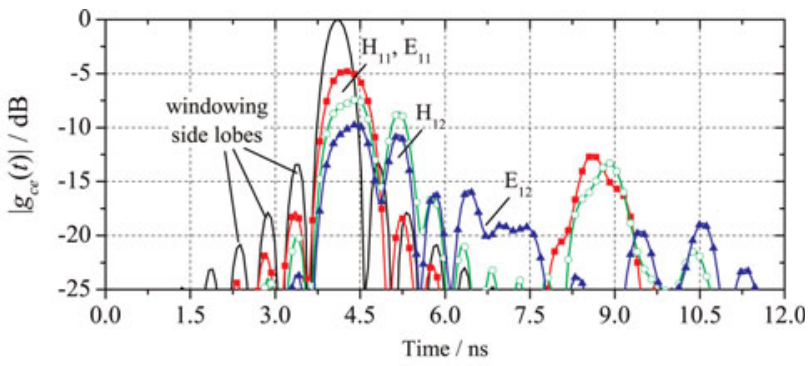

(b)

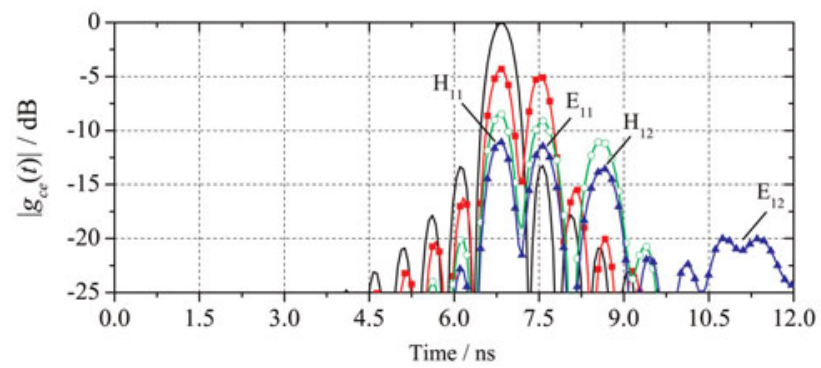

(c)

Fig. 5. Impulse response envelopes for the four simulated gauging scenarios at three reflector distances: (a) $l_{\text {refl }}=0.2 \mathrm{~m}$, (b) $l_{\text {refl }}=0.6 \mathrm{~m}$, and, (c) $l_{\text {refl }}=1.0 \mathrm{~m}$.

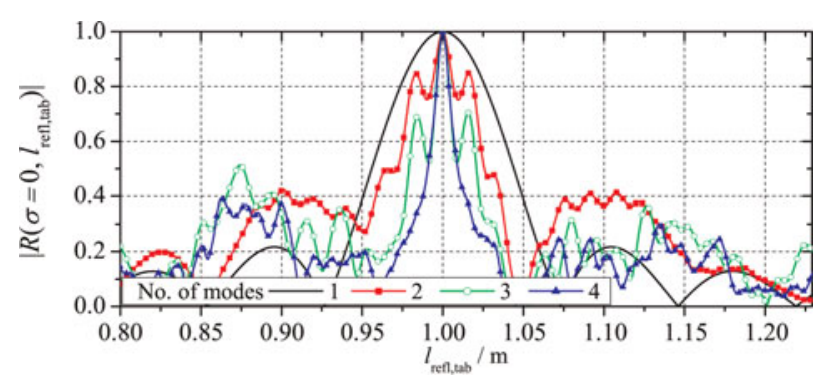

Fig. 6. $\left|R\left(\sigma=0, l_{\text {refl,tab }}\right)\right|$ at $l_{\text {refl }}=1.0 \mathrm{~m}$.

Because in this case simulation data is correlated with simulation data, the measurement error would be always zero because at $l_{\text {refl }}=l_{\text {refl, tab }}$ the "simulated measurement signal" and the reference signal are identical. However, if $l_{\text {refl }}$ is not resolved exactly within all available $l_{\text {refl, tab }}$, a residual distance error in the order of the step width $\Delta l_{\text {refl,tab }}$ occurs. Additionally, if $\Delta l_{\text {refl,tab }}$ is chosen to large, a small correlation peak might not be resolved, which might lead to a false detection on side lobe maxima also resulting in measurement errors.

Moreover, Fig. 7 depicts the progression of $\left|R\left(\sigma=0, l_{\text {ref }}\right)\right|$ for all scenarios in dependence of $l_{\text {refl,tab }}$ and, in contrast to
Fig. 6, additionally in dependence of the distance $l_{\text {refl }}$. The included graphs thus represent the whole dataset, which has to be evaluated for depicting a measurement error curve over the whole distance interval of $l_{\text {refl }}=\left[\begin{array}{lll}0 & \ldots & l_{\text {refl, max }}\end{array}\right]$. Consequently, a horizontal line corresponds to an illustration in terms of Fig. 6. Because maximal correlation is expected at $l_{\text {refl }}=l_{\text {refl,tab }}$, the maximum always occurs on the bisecting line of the quadrant, i.e. at an angle of $45^{\circ}$ to the $x$-axis.

Figure 7 additionally confirms the tendency to narrow maximum main and side lobes with increasing signal complexity, i.e. with a rising number of modes. In case of two to four modes strong side lobes appear at an angle of $\arctan (1 / 2) \approx$ $26.6^{\circ}$ and $\arctan (2 / 1) \approx 63.4^{\circ}$ to the $x$-axis. In this case, the main pulse package from the reference data coincides with the first replica of the measurement data or vice versa.

\section{B. Investigations on reliability and accuracy}

The correlation-based algorithm in this contribution depends on accurate modeling and simulation of the real measurement setup to provide adequate reference simulation data for comparison. Thus, in this section, investigations are made on how intensive deviations between simulation and reality are affecting the functionality, reliability, and accuracy of the proposed

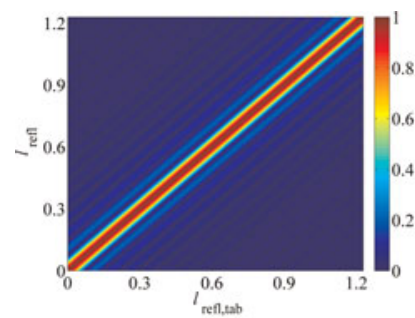

(a)

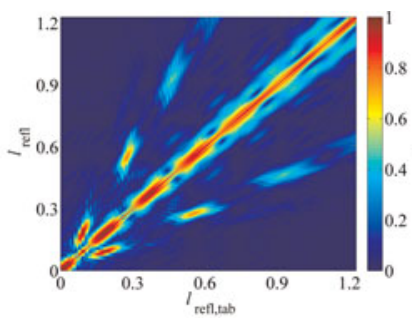

(b)

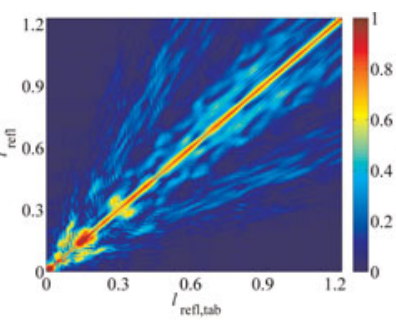

(c)

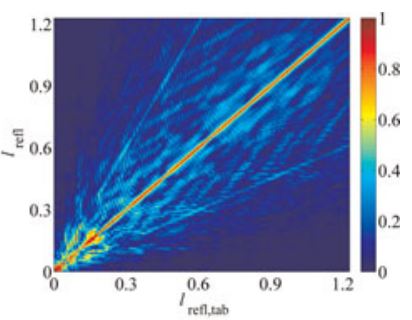

(d)

Fig. 7. $\left|R\left(\sigma=0, l_{\text {refl, tab }}\right)\right|$ for all $l_{\text {refl, tab }}$ and $l_{\text {refl }}$ in case of one to four modes: (a) one mode, (b) two modes, (c) three modes and, (d) four modes. 
measurement method in dependence of the applied mode variety or the signal complexity, respectively.

In contrast to deviations like e.g. length offsets, which might be compensated via signal shifting prior to correlation, the worst case for the proposed method are unconformities, which affect the distance-dependent shape of the signal. Thus, this section analyzes the deviation of the inner tube diameter $d_{s p}$, i.e. the consequences of assuming a too large or too small tube cross-section in the simulation in comparison to the measurement setup. If the diameter deviations are relatively small, the mode excitation levels within the tube remain almost unchanged, so the mode power distribution is not modified for the following investigations. As the most serious consequence, changes of the inner diameter directly affect the modes' cut-off frequencies and thus their propagation properties and the effects of intermodal dispersion.

To illustrate the impact of this matter, Fig. 8 compares the impulse response envelope in case of the four mode scenario with $d_{s p}=84.9 \mathrm{~mm}$ and $l_{\text {refl }}=1.0 \mathrm{~m}$ to the ones occurring in presence of diameter discrepancies of $\Delta d= \pm 0.5 \mathrm{~mm}$. Especially signal portions arriving late and having thus traveled long inside the tube, e.g. signal portions of modes with high cut-off frequencies or multiple pulse replicas, exhibit noticeable variances in magnitude, shape and position, compared to the original signal.

The longer a signal travels inside the simulated tube of a "wrong" diameter, the worse the discrepancies to the measured signals are. Consequently, similarity between the signals decreases with increasing reflector distance $l_{\text {refl }}$, too. Modes with higher cut-off frequencies are more affected because their propagation velocity or their propagation constant $\beta$, respectively, changes more severe with $\Delta d$. As a result, the longer the tube is, the smaller the deviations have to be in order to still achieve suitable reference data.

Being now aware of the deviations' qualitative properties, a quantitative conjunction is made in Fig. 9 between the diameter discrepancies and the resulting maximal error magnitude $\left|e_{\max }\right|$, for correlation being done with inaccurate reference signals emerging from $\Delta d$. Obviously for the one and the two mode scenario the measurement error increases more or less linear within the observed distance interval of $l_{\text {refl }}=$ $\left[\begin{array}{lll}0 & \ldots & l_{\text {ref, } \max }\end{array}\right]$, whereas in case of three modes the error jumps up at $|\Delta d| \approx 0.3 \mathrm{~mm}$ to a higher level of about $\left|e_{\max }\right|=15 \mathrm{~mm}$ being representative for the algorithm unlatching on a "wrong" maximum. As expected, for the four mode scenario, which exhibits the strongest diameter dependence as discussed before, the unlatching occurs even earlier at $|\Delta d| \approx 0.15 \mathrm{~mm}$. If $|\Delta d|$ is increased more, the maximal error for four modes escalates to another higher error level, whereas the three mode scenario still remains at

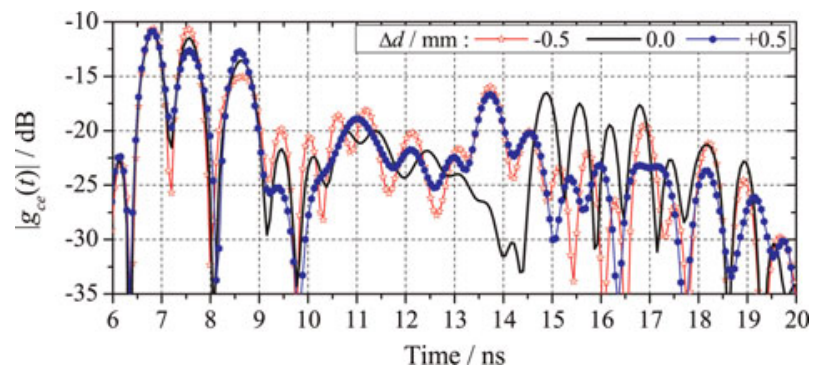

Fig. 8. Envelopes in case of diameter deviations for $l_{\text {refl }}=1.0 \mathrm{~m}$.

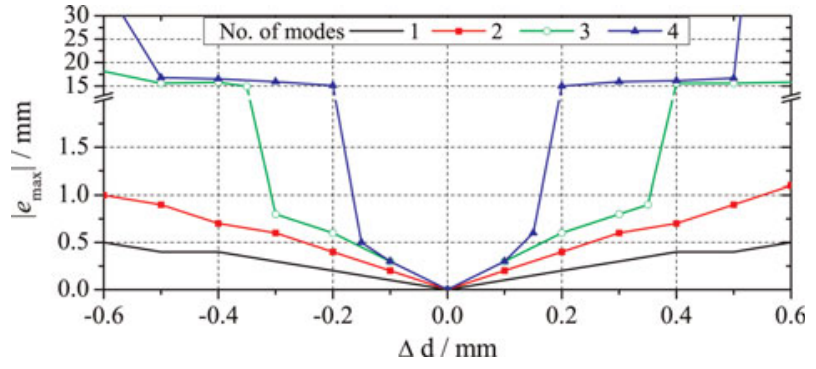

Fig. 9. Maximal error magnitude $\left|e_{\max }\right|$ over $\Delta d$.

about $\left|e_{\max }\right|=15 \mathrm{~mm}$. This clarifies the strongest sensitivity of four modes against diameter deviations.

Figure 10 investigates the unlatching behavior in detail by depicting $\left|R\left(\sigma=0, l_{\text {refl,tab }}\right)\right|$ at $l_{\text {refl }}=1.0 \mathrm{~m}$ in case of $\Delta d=-0.5 \mathrm{~mm}$ for the four mode scenario. For this reflector distance the discrepancies between the "simulated measurement" signal and the reference data in case of three and four modes are already crucial. This results in a global maximum of $\left|R\left(\sigma=0, l_{\text {ref, }, \text { ab }}\right)\right|$ not occurring at the expected correct position $l_{\text {refl }}=l_{\text {ref, tab }}$ anymore, leading the algorithm to unlatch on a "wrong" peak nearby. In the scenarios of one or two modes the algorithm is not yet unlatched, as those scenarios are less sensitive to diameter deviations.

In Fig. 11 the effect of evolving side maxima within $\mid R(\sigma=$ $\left.0, l_{\text {reff,tab }}\right) \mid$ is accentuated for $\Delta d=-0.5 \mathrm{~mm}$. The solid curves represent the global maximum of $\left|R\left(\sigma, l_{\text {refl,tab }}\right)\right| \forall l_{\text {reff, tab }}$, which is detected and evaluated by the algorithm, in dependence of the distance $l_{\text {reff }}$, which is to be measured. In addition, $\left|R\left(\sigma=0, l_{\text {refl,tab }}=l_{\text {reff }}\right)\right|$ is plotted as a dotted curve, which represents the values on the $45^{\circ}$-lines in Fig. 7 , where the global maximum in case of perfect conformance between measurement and reference signal is anticipated to be (only observable here via simulation with the knowledge of the actual reflector

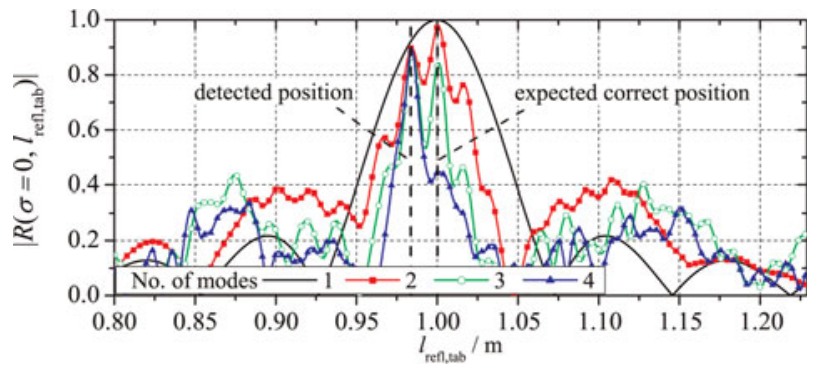

Fig. 10. $\left|R\left(\sigma=0, l_{\text {reff,tab }}\right)\right|$ for $l_{\text {refl }}=1.0 \mathrm{~m}$ and $\Delta d=-0.5 \mathrm{~mm}$.

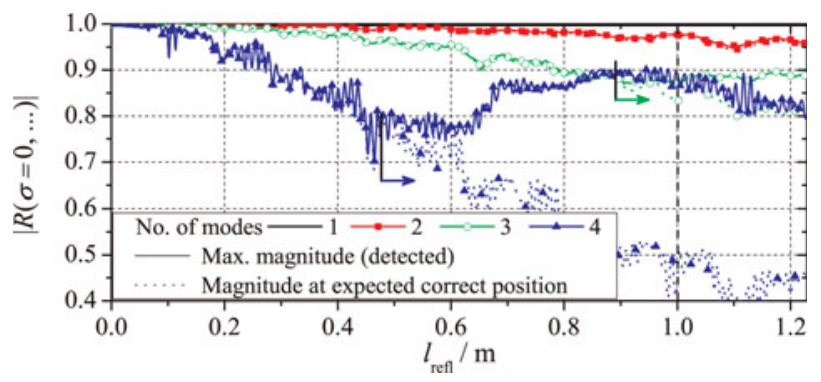

Fig. 11. Maximum of $\left|R\left(\sigma, l_{\text {reff,tab }}\right)\right| \forall l_{\text {reff,tab }}$ and $\left|R\left(\sigma=0, l_{\text {ref }}=l_{\text {ref } f}\right)\right|$ in case of $\Delta d=-0.5 \mathrm{~mm}$. 


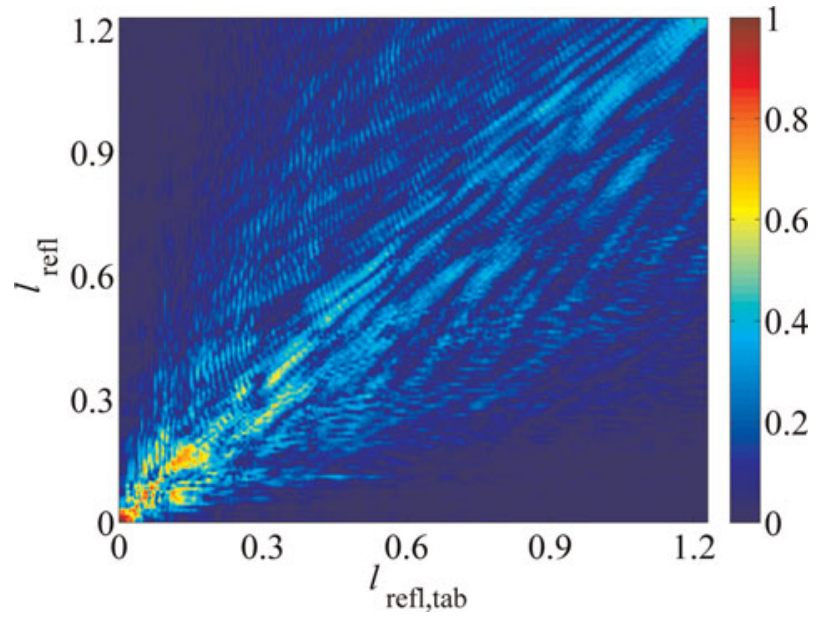

Fig. 12. $\left|R\left(\sigma=0, l_{\text {refl,tab }}\right)\right|$ for all $l_{\text {refl,tab }}$ and $l_{\text {refl }}$ in case of four modes with $\Delta d=-5.0 \mathrm{~mm}$

distance). Consequently, whenever the solid and the dotted curves depart from each other, the global maximum takes place elsewhere than at the expected correct position $l_{\text {refl, } t a b}=$ $l_{\text {refl, }}$, thus causing measurement errors.

For a mode variety of one or two modes, the particular solid and dotted curves coincide widely, implying no significant measurement error to occur. For three or four modes the respective curves are diverging, starting from the marked particular positions of $l_{\text {refl }}$, forcing the algorithm to detect the inadequate maximum. This moment of unlatching occurs later in case of three modes, once again proving the fact of better robustness with decreasing mode variety. The magnitudes on the marked positions of Fig. 10 can be recognized at the dashed line for $l_{\text {refl }}=1.0 \mathrm{~m}$, making clear that the algorithm is not yet unlatched in the one or two mode scenario.

Additionally, Fig. 12 depicts $\left|R\left(\sigma=0, l_{\text {refl,tab }}\right)\right|$ over all measurement and reference distances (compare Fig. 7) in case of an even more deviant diameter with $\Delta d=-5.0 \mathrm{~mm}$. In this case no significant magnitude is observable on the bisecting line at all, deteriorating the measurement accuracy seriously.

\section{MEASUREMENT RESULTS}

In this section, the proposed method is used for measurements with different types of manufactured waveguide transition prototypes. The transitions are chosen to exhibit mode excitation levels being comparable to the investigated simulation scenarios used in the sections before.

On the one hand, the measurements are realized with a mechanically complex, obstacle-based transition (Fig. 17(c)) providing a mode-preserving transmission of the fundamental mode $H_{11}$ from the feeding to the large tube cross-section, thus exhibiting a similar mode excitation to the one mode scenario, avoiding multimode propagation. On the other hand, the same transition is used without its obstacle as a parabolic taper (Fig. 17(b)) with increased excitation of higher-order modes (see [4] for details and scattering parameters). Additionally, to account for the four mode scenario, and thus for the excitation of a variety of higher-order modes with significant amplitudes, a simple stepped waveguide transition is applied (Fig. 17(a)). Figure 13 depicts the waveguide transition as a $3 \mathrm{D}$ FIT model with its electric field distribution. The transition with a diameter step from the feeding waveguide with $d_{1}=22 \mathrm{~mm}$ to the overmoded tube diameter of $d_{s p}=84.9 \mathrm{~mm}$ basically excites the modes $H_{11}, E_{11}, H_{12}, E_{12}, H_{13}$ with excitation levels $\mid S_{2 \text { (Mode }}$ $x)_{1}\left(T E M / H_{11}\right) \mid$ of about -5 to $-10 \mathrm{~dB}$, if propagable (Fig. 14).

For this transition in Fig. 15 a comparison is made between a measured envelope $\left|g_{c e, \text { meas }}(t)\right|$ and a simulated reference one $\left|g_{c e}\left(t, l_{\text {refl,tab }}\right)\right|$ for a distance of $l_{\text {refl }}=l_{\text {refl,tab }}=1.0 \mathrm{~m}$. A good agreement between both curves exists, which underlines the realistic simulation of the measurement setup.

Figure 16 depicts the absolute values of the crosscorrelation function $\left|R\left(\sigma=0, l_{\text {refl, tab }}\right)\right|$ at an exemplary reflector distance in the setup of $l_{\text {refl }}=1.0 \mathrm{~m}$. Thus, for a reference distance $l_{\text {refl,tab }}$ coinciding with the actual distance $l_{\text {refl }}$ in the
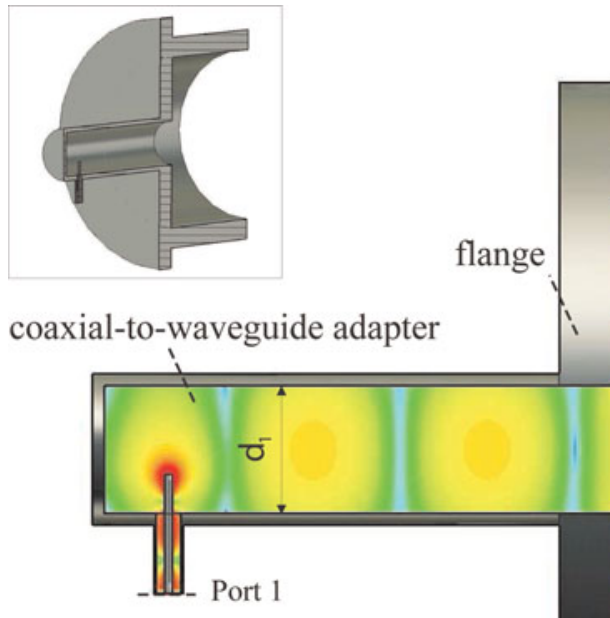

waveguide / metal tube
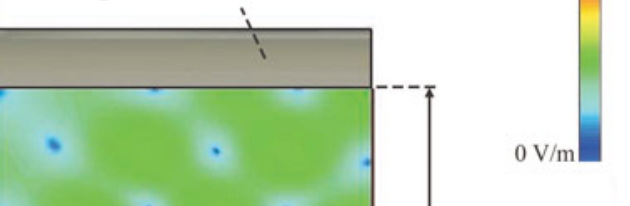

Fig. 13. Electric field inside the stepped transition (E-plane). 


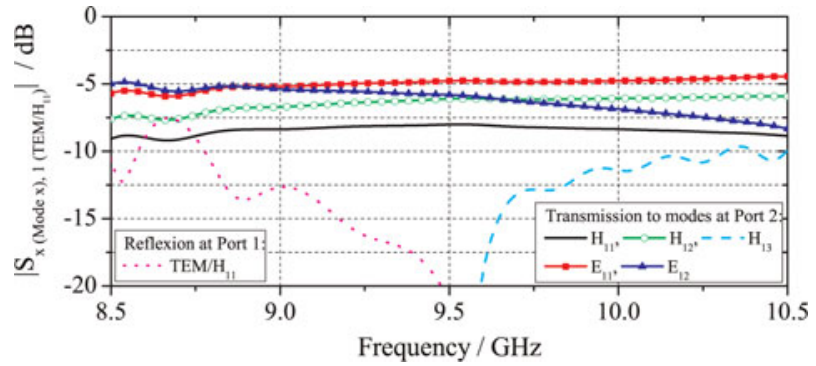

Fig. 14. Scattering parameters of the stepped transition.

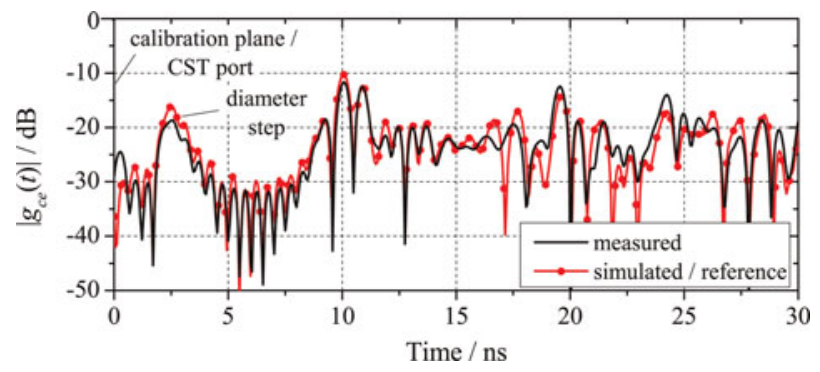

Fig. 15. Measured and simulated envelopes for $l_{\text {refl }}=l_{\text {refl,tab }}=1.0 \mathrm{~m}$.

setup, correlation is high and the theoretical maximum of $\left|R\left(\sigma=0, l_{\text {refl, tab }}\right)\right|$ is almost reached.

The maximal value for the stepped transition is slightly lower than in case of the other transitions because deviations between simulation and measurement setup carry more weight in case of multimode propagation due to signal portions cycling through the system more often (compare Section III.B). Moreover, the obtained peak in case of the stepped transition is narrower than the other ones. Additionally, the peak of the mode-preserving transition is moderately wider than the one for the parabolic transition and exhibits a lower level of side lobes, thus confirming the results from Section III.A.

To verify the capability of the proposed correlation-based method in terms of accuracy, the resulting measurement error for the transitions is depicted in Fig. 18, whereas a step width of $\Delta l_{\text {reff } t a b}=0.2 \mathrm{~mm}$ for the reference table is used. This leads to a vertical error curve resolution of $0.1 \mathrm{~mm}$. In case of the mode-preserving transition the curve exhibits a slightly stronger oscillating progression than the other ones, whereas a marginally inclining progression for all transitions is present, which is attributed to manufacturing tolerances like the deviation of the inner tube diameter. As desired, in all cases a continuous submillimeter accuracy over the total measurement range can be maintained. The measurement results therefore indicate, that despite the multimode propagation a submillimeter

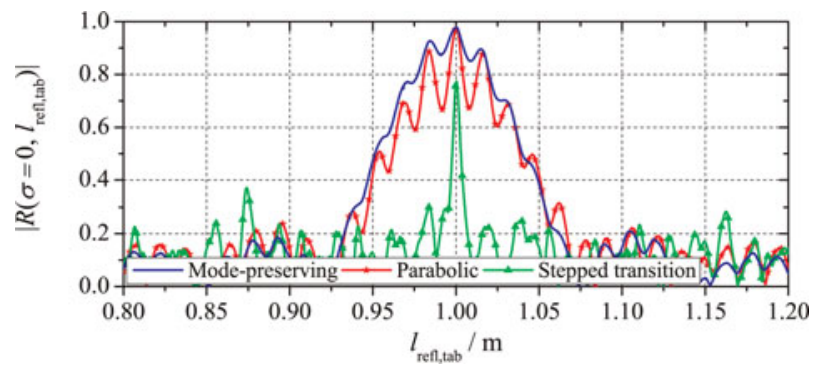

Fig. 16. $\left|R\left(\sigma=0, l_{\text {reft, tab }}\right)\right|$ at $l_{\text {refl }}=1.0 \mathrm{~m}$ for three different transitions.

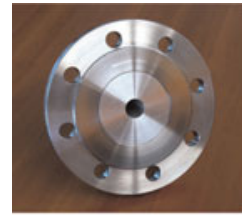

(a)

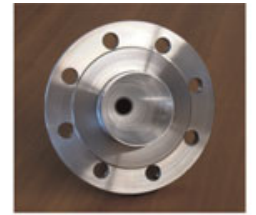

(b)

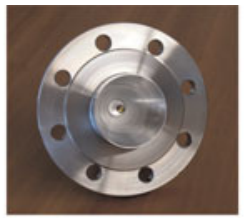

(c)
Fig. 17. Fabricated waveguide transitions: (a) stepped waveguide transition, (b) parabolic waveguide transition, and (c) mode-preserving waveguide transition.

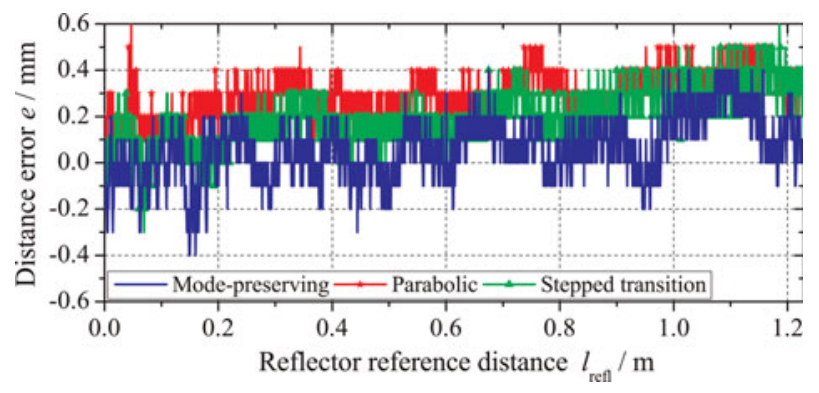

Fig. 18. Measurement results for correlation-based signal processing.

accuracy can be achieved, when waveguide transition and signal processing algorithm harmonize.

\section{CONCLUSIONS}

Parasitic intermodal dispersion is revealed as the main distortion effect of guided radar distance measurements conducted in large overmoded metal tubes, if conventional free-space optimized signal processing is applied. This contribution reviews the multimode propagation effects within the waveguide to derive a solution for overcoming the drawbacks of this scenario. An alternative correlation-based processing method is proposed, offering more degrees of freedom and even simplification in the design of waveguide transitions applied to the metal tube. In this way it is shown that multimode propagation can be exploited to achieve the desired accuracy. Considerations on the mode variety and its influence on the signal complexity as well as investigations on the technique's reliability and precision in case of mechanical tolerances like waveguide diameter deviations are presented. Consequently, the trade-off between the complexity of the signal processing algorithm and thus its computing intensity on the one hand and on the other hand the expenditure for the waveguide transition is clarified. The accomplished submillimeter accuracy is validated by measurements.

\section{REFERENCES}

[1] Brumbi, D.: Measuring process and storage tank level with radar technology, in Record of the IEEE 1995 Int. Radar Conf., May 1995, 256-260.

[2] Armbrecht, G.; Denicke, E.; Rolfes, I.; Pohl, N.; Musch, T.; Schiek, B.: Compact mode-matched excitation structures for radar distance measurements in overmoded circular waveguides. Ad. Radio Sci., 6 (2008), 9-17. Available Online at http://www.adv-radio-sci.net/6/9/ 2008/ 
[3] Pohl, N.; Gerding, M.; Will, B.; Musch, T.; Hausner, J.; Schiek, B.: High precision radar distance measurements in overmoded circular waveguides. IEEE Trans. Microw. Theory Tech., 55 (6) (June 2007), 1374-1381.

[4] Armbrecht, G.; Denicke, E.; Pohl, N.; Musch, T.; Rolfes, I.: Obstacle based concept for compact mode-preserving waveguide transitions for highprecision radar level measurements, in 38th European Microwave Conf. EuMC 2008, October 2008, 472-475.

[5] Denicke, E.; Armbrecht, G.; Rolfes, I.: A correlation-based method for precise radar distance measurements in dispersive waveguides, in 6th European Radar Conf. EuRAD 2009, September/October 2009, 302-305.

[6] Fernandez Casares, S.; Balle, S.; Menendez-Valdes, P.: Mode beating and spontaneous emission noise effects in a variable-waveguide model for the dynamics of gain-guided semiconductor laser arrays. IEEE J. Quantum Electron., 30 (11) (November 1994), 2449-2457.

[7] Marcuse, D.: Theory of Dielectric Optical Waveguides, 2nd ed., ser. Quantum Electronics - Principles and Applications, Academic Press Boston, 1991.

[8] Liu, M.; Shum, P.: Effects of intermodal dispersion on short pulse propagation in an active nonlinear two-core fiber coupler. IEEE Photon. Technol. Lett., 16 (4) (April 2004), 1080-1082.

[9] Skolnik, M.: Radar Handbook, McGraw-Hill New York, 2008.

[10] Roberts, M.J.: Signals and Systems: Analysis Using Transform Methods and MATLAB, McGraw-Hill New York, 2004.

[11] Tang, C.: Mode conversion in tapered waveguides at and near cutoff. IEEE Trans. Microw. Theory Tech., 14 (5) (1966), 233-239.

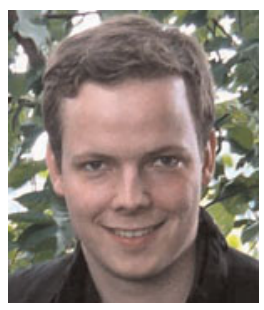

Eckhard Denicke was born in Hannover, Germany, in 1982. He received the Dipl.-Ing. degree in electrical engineering from the Leibniz Universität Hannover in January 2008. Since then, he has been a research assistant at the Institute of Radiofrequency and Microwave Engineering at the Leibniz Universität Hannover. His research interest is focused on radar applications, e.g. for industrial level measurements including the design of antenna and waveguide components as well as signal processing issues. Further fields of activity are ultra-wideband (UWB) communication systems and the electromagnetic compatibility (EMC) of aircrafts.

Mr. Denicke received the EADS Defence Electronics ARGUS Award 2008 and the VDE Student Award 2008 for his diploma thesis. Additionally, he was awarded with the third place in the AMTA Symposium 2009 Student Paper
Contest and received the EEEfCOM Innovation Award 2009 as member of a research consortium for contributions on "Advances in Industrial Radar Level Measurements".

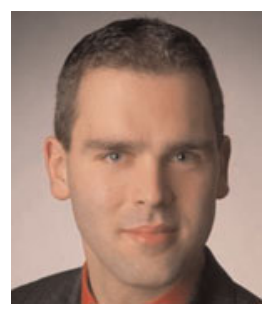

Gunnar Armbrecht was born in Seesen, Germany, in 1978. He received the Dipl.-Ing. degree in electrical engineering from the University of Hannover, Germany, in 2005. Since mid-2005, he has been a research assistant at the Institute of Radiofrequency and Microwave Engineering, Leibniz Universität Hannover, Germany. His current research interests include radar antenna design for industrial level measurement techniques, transient pulse propagation in GTEM cells and the design of compact ultra-wideband multiarm antennas for applications in multiple-inputmultiple-output (MIMO) transmission systems and diversity reception.

Mr. Armbrecht received the VDE Student Award for his diploma thesis in 2005 and the EEEfCOM Innovation Award for contributions on "Advances in Industrial Radar Level Measurements" as member of a research consortium in 2009 .

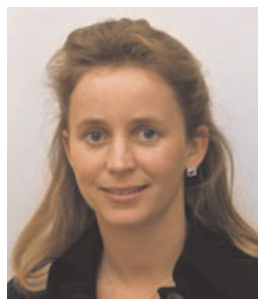

Ilona Rolfes was born in 1973 in Hagen, Germany. She received the Dipl.-Ing. and Dr.-Ing. degrees in electrical engineering from the Ruhr-University Bochum, Germany, in 1997 and 2002, respectively. From 1997 to 2005 she has been with the Research Group High Frequency Measurements at the Ruhr-University Bochum as a research assistant. From 2005 to 2009 she worked as a Junior professor in the department of electrical engineering at the Leibniz Universität Hannover, Germany, where in 2006 she became head of the Institute of Radiofrequency and Microwave Engineering. Since 2010 she leads the department for High Frequency Systems at the Ruhr-University Bochum. Her fields of research are concerned with high frequency measurement methods for vector network analysis, material characterization, noise characterization of microwave devices as well as sensor principles for radar systems and wireless solutions for communication systems. 